\title{
INJECTIVE MODULES OVER NON-ARTINIAN SERIAL RINGS
}

\author{
DAVID A. HILL
}

(Received 31 October 1985; revised 5 January 1987)

Communicated by R. Lidl

\begin{abstract}
A module is uniserial if its lattice of submodules is linearly ordered, and a ring $R$ is left serial if $R$ is a direct sum of uniserial left ideals. The following problem is considered. Suppose the injective hull of each simple left $R$-module is uniserial. When does this imply that the indecomposable injective left $R$-modules are uniserial? An affirmative answer is known when $R$ is commutative and when $R$ is Artinian. The following result is proved.

Let $R$ be a left serial ring and suppose that for each primitive idempotent e, eRe has indecomposable injective left modules uniserial. The following conditions are equivalent.

(a) The injective hull of each simple left $R$-module is uniserial.

(b) Every indecomposable injective left $R$-module is uniserial.

(c) Every finitely generated left $R$-module is serial.

The rest of the paper is devoted to a study of some non-Artinian serial rings which serve to illustrate this theorem.

1980 Mathematics subject classification (Amer. Math. Soc.): primary 16 A 52, 16 A 53; secondary 16 A 04.
\end{abstract}

\section{Introduction}

A module is said to be uniserial in case its submodules are linearly ordered by inclusion and it is serial if it is a direct sum of uniserial submodules. A ring $R$ is left serial if ${ }_{R} R$ is serial and $R$ is serial if it is both left and right serial. When $R$ is also Artinian a well-known theorem of Nakayama states that any module over $R$ is serial. For modules over non-Artinian serial rings Nakayama's theorem is in general false. However for finitely generated modules the theorem may still

(C) 1988 Australian Mathematical Society $0263-6115 / 88 \$ A 2.00+0.00$ 
be truse. For example if $R$ is left (right) Noetherian the finitely generated left (right) modules are serial [8].

Ivanov [4] has a theorem which links the structure of the finitely generated modules to the indecomposable injectives. His theorem states that the finitely generated left (right) $R$-modules are serial if and only if each indecomposable injective left (right) $R$-module is uniserial. It is an open question whether one can replace 'indecomposable injective' with 'injective hulls of simples' in Ivanov's theorem. Section 1 of this paper deals with this problem. The above question is reduced to the consideration of local rings (Proposition 1.5). An affirmative answer is given when $R$ is right (left) Noetherian and when $e R e$ is commutative for each primitive idempotent $e$.

Sections 2 and 3 are devoted to the construction of some classes of nonArtinian serial rings. These rings are shown to have finitely generated left and right modules serial if and only if $e R e$ is a complete discrete valuation ring for each primitive idempotent $e$.

All rings considered have an identity and all modules are unital. The Jacobson radical will be denoted by $J$, and for a given module $M$ the injective hull of $M$ will be denoted by $E_{R}(M)$ or simply $E(M)$ when $R$ is understood. The socle of $M$, defined as the largest semi-simple submodule of $M$, will be denoted by $S(M)$.

\section{Rings whose injective hulls of simple modules are uniserial}

In this section we consider the following problem: Let $R$ be a left serial ring whose injective hulls of simple left modules are uniserial. When does this imply that $R$ has indecomposable injective left modules uniserial? A solution to this problem is known to exist in two distinct cases: When $R$ is commutative [3] and when $R$ is left Artinian [2, Theorem 5.4].

We make the following definition: Let $R$ be a left serial ring and $R a$ a cyclic left $R$-module. $R a$ is said to be primitive in case there exists a primitive idempotent $e \in R$ such that $a=e a$. Thus $R a$ is primitive if and only if it is isomorphic to a factor module of a principal indecomposable left ideal.

1.1 Lemma. Assume $R$ is left serial and let $M$ be an indecomposable left $R$-module with $R a$ and $R b$ non-zero primitive submodules of $M$. Suppose there exists $K \subseteq J a$ such that $R a / K \subseteq R b / K$. Then $R a \subseteq R b$.

ProOF. By hypothesis there exists a primitive idempotent $e$ such that $a=e a$. Therefore, $a=\gamma b+k(\gamma \in e R, k \in e K)$. As $K \subseteq J a, k=x a$ for some $x \in e J e$. Therefore $(e-x) a=\gamma b$ and since $e-x$ is a unit in $e R e, R a \subseteq R b$. 
1.2 LEMMA. Let $R$ be a left serial ring whose injective hulls of simple left modules are uniserial and $M$ a left $R$-module generated by two primitive submodules $R a$ and $R b$ with $R a \cap R b \neq 0$. Then

(a) There exist submodules $R a_{1} \subseteq R a$ and $R b_{1} \subseteq R b$ such that $J a_{1}=J b_{1}=$ $J a \cap J b$.

(b) Suppose that $M$ is not uniserial. Consider $0 \neq c a \in J a \cap J b$. Then there exists a simple submodule $T$ of $\left(R a_{1}+R b_{1}\right) / J$ ca such that

$$
\left(R a_{1}+R b_{1}\right) / J c a=R a_{1} / J c a \oplus T=R b_{1} / J c a \oplus T .
$$

PrOOF. The case when $M$ is uniserial and therefore generated by $R a$ or $R b$ is trivial. So we will assume that $R a \nsubseteq R b$ and $R b \nsubseteq R a$. We first prove that for a given $0 \neq c a \in J a \cap J b$ statement (b) holds and then show that (a) is an immediate consequence of $(b)$.

Let $0 \neq c a \in J a \cap J b$ and consider $M / J c a$. Clearly $S(M / J c a) \neq 0$ and contains $S=R c a / J c a$. If $S(M / J c a)=S$ then by hypothesis $R a / J c a \subseteq R b / J c a$ or $R b / J c a \subseteq R a / J c a$ and by 1.1 this implies that $R a \subseteq R b$ or $R b \subseteq R a$. So we may assume that there exists a simple left $R$-module $T \subseteq S(M / J c a)$ such that $S \cap T=0$. Thus $T=(R(\mu a+\gamma b)+J c a) / J c a$ where $\mu, \gamma \in R$. Set $a_{1}=\mu a$, $b_{1}=\gamma b$ and consider the submodule $R a_{1}+R b_{1}$. Observe that the socle of $\left(R a_{1}+R b_{1}\right) / J c a$ also contains $S$ and $T$. Now $T \subseteq R a_{1} / J c a$ or $T \subseteq R b_{1} / J c a$ imply that $S=T$. Thus $T$ has zero intersection with $R a_{1} / J c a$ and $R b_{1} / J c a$. Therefore $R a_{1} / J c a \oplus T=R b_{1} / J c a \oplus T=\left(R a_{1}+R b_{1}\right) / J c a$ and (b) is proved for a given $c a$.

We observe that $a_{1}-k b_{1}+t \in J c a$ for some $k \in R$ and $t+J c a \in T$. Let $x \in J$. Then $x a_{1}-x k b_{1} \in J c a$. Since $J c a \subseteq J a \cap J b \subseteq J b_{1}$, we obtain $J a_{1} \subseteq J b_{1}$. Using a symmetric argument yields $J a_{1}=J b_{1}=J a \cap J b$. In order to prove (a) and (b) we need only note that using a different value of $c a$ in the above construction still yields the same values for $R a_{1}$ and $R b_{1}$.

1.3 Proposition. Suppose $R$ is a left uniserial ring with $J \neq J^{2}$. Then the left injective hull of $R / J$ is uniserial if and only if each indecomposable injective left $R$-module is uniserial.

ProOF. In one direction the result is obvious. So assume that the injective hull of ${ }_{R}(R / J)$ is uniserial. First observe that $R / J^{2}$ satisfies the same hypothesis as $R$ since $E_{R / J^{2}}\left(R / J^{2}\right) \subseteq E_{R}\left(J / J^{2}\right)$. So $R / J^{2}$ is an Artinian left uniserial ring whose injective hulls of simple left modules are uniserial. Therefore applying [2, Theorem 5.4] $R / J^{2}$ is uniserial. This implies that $J / J^{2}$ is cyclic both as a left and right $R$-module. Since $J^{2} \neq J, J^{2}$ is superfluous in $J$ and so $J$ is a cyclic left and right $R$-module. Using this fact an easy argument shows that there exists a $t \in J$ such that $R t=t R=J$. Now let $E$ be an indecomposable injective 
left $R$-module and $R a$ and $R b$ non-zero submodules of $E$. Applying 1.2 we may assume that $J a=J b=J a \cap J b$. Therefore $t a=\gamma b$ with $\gamma \in J$. Thus $t a=t \alpha b$ for some $\alpha \in R$. So $t(a-\alpha b)=0$ which implies that $J(a-\alpha b)=0$. Therefore $a=\alpha b$ or $a-\alpha b \neq 0$ and $R(a-\alpha b)$ is a simple submodule of $E$. Either case yields that $E$ is uniserial.

Recall that a ring is said to be duo in case all the one sided ideals are two sided.

1.4 COROLlaRY. Suppose $R$ is a left uniserial ring whose left injective hull of $R / J$ is uniserial. If $R$ is left or right Noetherian then $R$ is a duo uniserial Noetherian ring whose indecomposable left and right injective modules are uniserial.

Proof. Applying 1.3 and [4] $R$ is a uniserial ring whose indecomposable injective left $R$-modules are uniserial. Since $R$ is left or right Noetherian a well known argument shows that $\cap J^{n}=0$ and that $R$ is a duo, Noetherian principal ideal ring. An application of $[4$, Theorem 2] yields that $R$ has indecomposable injective right modules uniserial.

1.5 Proposition. Suppose $R$ is left serial and injective hulls of simple left $R$-modules are uniserial. If for each primitive idempotent $e$, the ring eRe has indecomposable injective left modules uniserial then $R$ has indecomposable injective left modules uniserial.

ProOF. Let $E$ be an indecomposable injective left $R$-module with $R a$ and $R b$ non-zero primitive submodules of $E$ and $M=R a+R b$. We will suppose that $R a \nsubseteq R b$ and $R b \nsubseteq R a$ and show that this yields a contradiction. By virtue of 1.2 we may assume that $R a \cap R b=J a=J b$. The proof is divided into three separate cases.

Case 1. Let $e$ be a primitive idempotent and suppose that $a=e a, b=e b$ and $e R \cdot c a \neq 0$ for all non-zero $c a \in J a$. Consider the $e R e$-module $e M$. Then $e J e a$ is essential in $e M$. For consider $e T \neq 0, e T \subseteq e M$. Thus $R e T \cap J a \neq 0$. Therefore there exists $c a \in R e T \cap J a$ such that $e$ Reca $\neq 0$. This means that $e T \cap e J a \neq 0$. Thus $E_{e R e}(e J a)=E_{e R e}(e M)$. Since $e J a$ is uniserial $E_{e R e}(e J a)$ is indecomposable. So by hypothesis $E_{e R e}(e M)$ is uniserial. Therefore $e a \in e R e b$ or $e b \in e R e a$. In either case $R a \subseteq R b$ or $R b \subseteq R a$.

Case 2. We suppose that $a=e a, b=e b$ and there exists a non-zero submodule $K \subseteq J a$ such that $e K=0$. Consider the set of all non-zero elements of $K$ indexed by a set $A$. For each $\alpha \in A$ let $M_{\alpha}=M / J c_{\alpha} a$. If for some $\alpha, S\left(M_{\alpha}\right)=$ $R c_{\alpha} a / J c_{\alpha} a$ we are through by 1.1 . So assume the contrary. In that case 1.2 
implies that for each $\alpha$ there exists a simple module $T_{\alpha}$ which is a direct summand of $M_{\alpha}$. Each $T_{\alpha}$ is generated by an element $a+\gamma_{\alpha} b$ modulo $J c_{\alpha} a$ where $\gamma_{\alpha}=e \gamma_{\alpha}$. So consider the set $\left\{a+\gamma_{\alpha} b\right\}(\alpha \in A)$. First observe that for $R c_{\beta} a \subseteq R c_{\alpha} a$ the image $T_{\beta}^{\prime}$ of $T_{\beta}$ under the natural homomorphism of $M_{\beta}$ to $M_{\alpha}$ is a non-zero simple direct summand of $M_{\alpha}$. Therefore $M_{\alpha}=T_{\alpha} \oplus T_{\beta}^{\prime}$ or $T_{\alpha}=T_{\beta}^{\prime}$. The first case cannot occur since it would imply that $R a \cap R b=J c_{\alpha} a$. This would mean that $R c_{\alpha} a=R a$ a contradiction. So there exists a unit $\mu_{\alpha \beta} \in e R e$ such that $a+\gamma_{\alpha} b=\mu_{\alpha \beta}\left(a+\gamma_{\beta} b\right)$ modulo $J c_{\alpha} a$. That is

$$
a+\gamma_{\alpha} b-\mu_{\alpha \beta}\left(a+\gamma_{\beta} b\right)=x c_{\alpha} a \quad \text { for some } x \in J \text {. }
$$

But $e x c_{\alpha} a=0$ so that $a+\gamma_{\alpha} b=\mu_{\alpha \beta}\left(a+\gamma_{\beta} b\right)$. Therefore $R\left(a+\gamma_{\alpha} b\right)=R\left(a+\gamma_{\beta} b\right)$ for all $\alpha, \beta \in A$. Setting $T=R\left(a+\gamma_{\alpha} b\right)$ yields for each $\alpha \in A, T \cap R a \subseteq J c_{\alpha} a$. Now $\bigcap R c_{\alpha} a$ is simple or zero. If the intersection is simple apply the hypothesis. If it is zero then $T \cap R a=0$ a contradiction as $E$ would then contain two non-zero submodules with intersection zero.

Case 3. Suppose that $a=e a$ and $b=f b$ with $e$ and $f$ primitive orthogonal idempotents such that $R e \not R f$. We may assume that $J a$ is not zero and choose $0 \neq c a \in J a$. Applying 1.2(b) there exists a simple module $T$ such that $T$ is a direct summand of $M / J c a$ and $T \cap(R c a / J c a)=0$. As before $T$ is generated modulo $J c a$ by an element $t=a+\gamma b$. Obviously $\gamma b \notin J b$ since containment implies $T=R c a / J c a$. Therefore $R \gamma b=R b$. Since $f R a \subseteq J a$ we obtain $R t / J t \cong R b / J b \cong R f / J f$. Using a similar argument we also have $R t / J t \cong R a / J a \cong R e / J e$ a contradiction as $R e \not R f$. Thus in all cases $R a \subseteq R b$ of $R b \subseteq R a$.

Now suppose that $f$ is a primitive idempotent and $E=E(R f / J f)$. By a result of Roux [6, Lemma 3.4], $f E$ is the left $f R f$ injective hull of $f R f / f J f$. If $E$ is uniserial so is $f E$. Thus we have the following lemma.

1.6 LEMMA. Let $R$ be a left serial ring and suppose that the injective hull of each simple left module is uniserial. Then for each primitive idempotent $f$, the left injective hull of $f R f / f J f$ is uniserial.

REmarK. It is easy to see that Proposition 1.5 and Lemma 1.6 reduce the problem of when injective hulls of simples being uniserial implies that all indecomposable injectives are uniserial to the study of local rings. In fact one can say a little bit more. As a consequence of Proposition 1.3 one need only consider the problem for the case when $R$ is local and $J=J^{2}$.

1.7 THEOREM. Let $R$ be a left serial ring and suppose that for each primitive idempotent $e, e R e$ has indecomposable injective left modules uniserial. The following conditions are equivalent. 
(a) The injective hull of each simple left $R$-module is uniserial.

(b) Every indecomposable injective left $R$-module is uniserial.

(c) Every finitely generated left $R$-module is serial. Under the above conditions $R$ is a serial ring.

Proof. The last statement and the equivalence of (b) and (c) is just [4, Theorem 2]. For (a) implies (b) apply 1.5. That (b) implies (a) is trivial.

1.8 COROLLARY. Let $R$ be a left serial ring and suppose that for each primitive idempotent $e$, eRe is commutative. Then conditions (a), (b), and (c) of 1.7 are equivalent.

PrOOF. It suffices to show that (a) implies the other two conditions. If (a) holds apply 1.6 and [3, Theorem, page 142] to get that $e R e$ has indecomposable injectives uniserial for $e$ a primitive idempotent. Now apply 1.7 .

1.9 COROLLARY. Suppose $R$ is a left or right Noetherian ring. Then conditions (a), (b), and (c) of Theorem 1.7 are equivalent.

ProOF. Apply 1.3, 1.6, and 1.7.

\section{Indecomposable injective modules over a class of non-Noetherian serial rings}

A discrete valuation domain $A$ (not necessarily commutative) is a uniserial, Noetherian integral domain with a non-zero radical. $A$ is said to be complete if it is complete with respect to its $J$-adic topology. Let $Q$ be the skew field of quotients of $A$, and $R$ be the ring of matrices of the form

$$
R=\left[\begin{array}{ll}
A & Q \\
0 & Q
\end{array}\right]
$$

where addition and multiplication are just addition and multiplication for matrices. So $R$ is a serial, right Noetherian, right hereditary ring but is not left Noetherian. This section is devoted to describing the indecomposable injective left $R$-modules. We will let $\hat{A}$ denote the completion of $A$, and $\hat{Q}$ the skew field of quotients of $\hat{A}$. Set

$$
\hat{R}=\left[\begin{array}{cc}
\hat{A} & \hat{Q} \\
0 & \hat{Q}
\end{array}\right]
$$

Note that $\hat{Q} / J(\hat{A})^{n} \cong Q / J(A)^{n}$ canonically for all positive integers $n$. 
2.1 Proposition. The following conditions are equivalent for a discrete valuation domain $A$ with skew field of quotients $Q$.

(a) $A$ is complete.

(b) For any pair of $A$-submodules $I$ and $K$ of $Q$ and homomorphism $\varphi \in$ $\operatorname{Hom}_{A}(Q / I, Q / K)$, there exists $d \in Q$ with $I d \subseteq K$ such that $\varphi(q+I)=q d+K$ for all $q+I \in Q / I$.

(c) $A \cong \operatorname{End}_{A}(Q / A)$.

If $K=0$, statement (b) is true without the hypothesis of completeness.

Proof. (a) implies (b). Let $I$ and $K$ be left $A$-submodules of $Q$ and $\varphi \in$ $\operatorname{Hom}_{A}(Q / I, Q / K)$. Consider the following diagram with $\pi_{1}$ and $\pi_{2}$ the natural projections from $Q$ to $Q / I$ and $Q$ to $Q / K$ respectively.

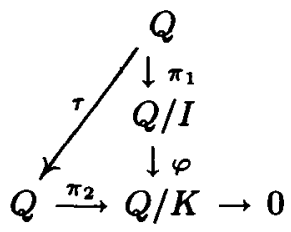

Now $A$ being complete is equivalent to $Q$ being quasi-projective over $A[7$, Theorem 8]. Therefore the map $\varphi \pi_{1}$ can be extended to a homomorphism $\tau$ which is given as a right multiplication by an element $d \in Q$. Let $q \in Q$. Then

$$
\varphi \pi_{1}(q)=\varphi(q+I)=\pi_{2} \tau(q)=q d+K .
$$

It is clear that $I d \subseteq K$. (b) implies (c). Take $I=K=A$. Thus $A d \subseteq A$ implies that $d \in A$. The rest is trivial. (c) implies (a). This is immediate and is left to the reader. The last statement is also straightforward and is left to the reader.

We will need the following concept: A module $N$ is said to be injective relative to $M$ in case for every submodule $K$ of $M$ and homomorphism $\delta: K \rightarrow N$ there is an extension of $\delta$ to $M$. When $M=N, N$ is said to be quasi-injective.

2.2 LeMMA. Let $R$ be a ring and $M$ an $R$-module. Suppose that $M / K$ is quasi-injective and has the property that for any submodule $L$ of $M$ and non-zero homomorphism $f: L \rightarrow M / K, K$ is contained in the kernel of $f$. Then $M / K$ is injective relative to $M$.

ProOF. Using the hypothesis $f$ factors to a homomorphism

$$
f^{\prime}: L / K \rightarrow M / K \text {. }
$$

Since $M / K$ is quasi-injective $f^{\prime}$ can be extended to $f^{\prime \prime}: M / K \rightarrow M / K$. Set $h=f^{\prime \prime} \pi$ where $\pi$ is the canonical projection of $M$ to $M / K$. A straightforward computation shows that $h$ extends $f$. 
2.3 THEOREM. Let $R=\left[\begin{array}{ll}A & Q \\ 0 & Q\end{array}\right]$. The indecomposable injective left $R$-modules are of the following three types.

(a) $E_{0}=\left[\begin{array}{l}Q \\ Q\end{array}\right]$.

(b) $E_{1}=\hat{E}_{0} /\left[\begin{array}{l}\hat{A} \\ 0\end{array}\right]$ where $\hat{E}_{0}=\left[\begin{array}{l}\hat{Q} \\ \hat{Q}\end{array}\right]$.

(c) $E_{2}=E_{0} /\left[\begin{array}{c}Q \\ 0\end{array}\right]$.

Proof. Using that $\hat{Q} / \hat{A} \cong Q / A$ it is clear that all the modules in question are indecomposable. We need only show injectivity. An application of 2.2 shows that the modules of type (c) are injective. The proof for the type (a) case goes like the one for type (b) with suitable modifications, so we will give the proof for the type (b) case.

Consider $E_{1}$. We will first show that $E_{1}$ is injective as an $\hat{R}$-module relative to $\hat{E}_{0}$. Suppose there is an $\hat{R}$-homomorphism $\varphi: I \rightarrow E_{1}$ where $I$ is an $\hat{R}$ submodule of $\left[\begin{array}{l}\hat{Q} \\ 0\end{array}\right]$. Thus the image of $I$ is contained in $\left[\begin{array}{c}\hat{Q} / \hat{A} \\ 0\end{array}\right] \subseteq E_{1}$. Since $\left[\begin{array}{c}\hat{Q} / \hat{A} \\ 0\end{array}\right]$ is canonically $\hat{A}$-isomorphic to the $\hat{A}$-injective module $\hat{Q} / \hat{A}, \varphi$ can be extended to $\left[\begin{array}{l}\hat{Q} \\ 0\end{array}\right]$. So we need only consider the following diagram

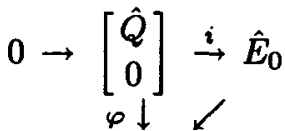

$$
\begin{aligned}
& E_{1}
\end{aligned}
$$

Since $\varphi$ is naturally an $\hat{A}$-homomorphism, apply 2.1 (b) to get that $\varphi$ is given by right multiplication in the first coordinate of $E_{1}$ modulo $\hat{A}$ by a fixed element $\hat{\alpha} \in \hat{Q}$. Extend $\varphi$ to the map $\varphi^{\prime}$ that carries a given $x=\left[\begin{array}{l}x_{1} \\ x_{2}\end{array}\right] \in \hat{E}_{0}$ to $\varphi^{\prime}(x)=$ $\left[\begin{array}{c}x_{1} \hat{\alpha}+\hat{A} \\ x_{2} \hat{\alpha}\end{array}\right] \in E_{1}$.

Now if $I$ contains $\left[\begin{array}{l}\hat{Q} \\ 0\end{array}\right]$ observe that $\varphi$ restricted to the second coordinate will be given by right multiplication by some $\hat{\alpha} \in \hat{Q}$ and that $\varphi$ restricted to [ $\left[\begin{array}{l}\hat{Q} \\ 0\end{array}\right]$ will be given by right multiplication by the same $\hat{\alpha}$ modulo $\hat{A}$. So extend $\varphi$ to $E_{1}$ using the same map as before.

Now a routine computation shows that any $R$-homomorphism from an $R$ submodule of $\hat{E}_{0}$ to $E_{1}$ can be extended to an $\hat{R}$-homomorphism on a suitable $\hat{R}$-submodule of $\hat{E}_{0}$. Therefore $E_{1}$ is injective relative to $\hat{E}_{0}$ as an $R$-module. This shows that $E_{1}$ is $R$-injective since $R$ can be embedded in 2 copies of $E_{0}$.

As a consequence of 2.3 it is easy to see that the indecomposable injective left $R$-modules are uniserial if and only if $A$ is complete. Thus by 1.7 we have the following corollary.

2.4 COROLlaRY. Let $R$ be as in 2.3. The following conditions are equivalent. 
(a) $A$ is complete.

(b) The injective hull of each simple left $R$-module is uniserial.

(c) Every indecomposable injective left $R$-module is uniserial.

(d) Every finitely generated left $R$-module is serial.

REMARK. Since $R$ is right Noetherian the right analogues of (b), (c), and (d) of 2.4 are always satisfied.

\section{The semi-direct product ring of a valuation domain by its ring of quotients}

Let $A$ be a ring and $V$ a two sided bimodule over $A$. Then $R=(A, V)$ is the semi-direct product ring of $V$ with multiplication defined by

$$
(a, x) \cdot\left(a^{\prime}, y\right)=\left(a a^{\prime}, a y+x a^{\prime}\right), \quad a, a^{\prime} \in A, x, y \in V .
$$

Thus $R$ is isomorphic to the ring of matrices of the form

$$
\left[\begin{array}{ll}
a & x \\
0 & a
\end{array}\right], \quad a \in A, x \in V
$$

with addition and multiplication being the obvious ones. We consider the case when $A$ is a commutative discrete valuation domain and $V=Q$ the ring of quotients of $A$. Thus $R=(A, Q)$ is a non-Noetherian commutative uniserial ring. Recall that a ring is said to be maximal if every family of pairwise solvable congruences of the form $x=x_{\alpha} \bmod \left(I_{\alpha}\right)$ has a simultaneous solution where $x_{\alpha} \in R$ and each $I_{\alpha}$ is an ideal of $R . R$ is called almost maximal if each of its proper homomorphic images is maximal.

3.1 Proposition. The ring $R=(A, Q)$ has every indecomposable injective module uniserial if and only if $A$ is complete.

Proof. Consider all the factor rings of $R$ with a non-zero socle. They are either Artinian or are of the form $(A, E), E \cong E(A / J(A))$. Applying [1, Corollary 6.B] these rings are self injective if and only if $A$ is complete. An easy consequence of the results of [3] shows that these rings are self injective if and only if they are maximal which is the case if and only if $R$ is almost maximal. Now applying [3, Theorem, page 142] shows that this is the case if and only if $R$ has every indecomposable injective uniserial.

REMARKS. (1) The class of rings characterized in 3.1 yields commutative uniserial rings whose indecomposable injective modules are not all uniserial by taking $A$ not complete. 
(2) Since $E(A / J(A)) \cong Q / A$, the rings of Faith [1, Corollary 6.B] are just the factor rings of the rings in 3.1 which have a non-zero socle.

(3) If $A$ is complete, Proposition 3.1 gives examples of uniserial rings whose finitely generated modules are serial but which satisfy no chain conditions.

\section{References}

[1] C. Faith, 'Self injective rings', Proc. Amer. Math. Soc. 77 (2) (1979), 157-164.

[2] K. R. Fuller, 'On indecomposable injectives over Artinian rings', Pacific J. Math. 29 (1969), 115-135.

[3] D. T. Gill, 'Almost maximal valuation rings', J. London Math. Soc. (1) 4 (1971), 140-146.

[4] G. Ivanov, 'Decomposition of modules over serial rings', Comm. Algebra 3 (1975), 10311036.

[5] G. Ivanov, 'Left serial rings which are left Noetherian', unpublished.

[6] B. Roux, 'Sur la dualité de Morita', Tôhoku Math. J 23 (1971), 457-472.

[7] S. Singh, 'Quasi-injective and quasi-projective modules over hereditary Noetherian prime rings', Canad. J. Math. 26 (1974), 1173-1185.

[8] R. B. Warfield, Jr., 'Serial rings and finitely presented modules', J. Algebra 37 (1975), 187-222.

Instituto de Matematica

Universidade Federal de Bahia

Salvador, Bahia

Brasil 